\title{
Shape constancy in pictorial representation
}

\author{
HANS WALLACH and FREDERICK J. MARSHALL \\ Swarthmore College, Swarthmore, Pennyslvania
}

\begin{abstract}
When one looks at a picture from an oblique direction, its retinal projection is foreshortened, but this distortion in proximal stimulation is usually not perceived. A correction akin to shape constancy takes place. Experiments are reported that show that this correction can be the result of compensation that takes the slant of the picture plane relative to the line of sight into account. When deceptive cues for the orientation of the picture plane were provided, the perceived shape in the picture conformed to a compensation based on the deceptive cues.
\end{abstract}

The shape of a surface that is slanted in relation to the line of sight is often correctly perceived, although the shape of its retinal image is that of the projection of the surface on a plane orthogonal to the line of sight. This fact is called shape constancy. In his exhaustive treatment of shape constancy, Hochberg (1971) discussed two explanations. One explanation assumes that the shape of the surface is familiar, that is, that memory of its true shape has an influence on perception. The other explanation assumes that a compensation process of the sort that causes size constancy operates with the slant of the surface being taken into account. Although there is evidence that shape constancy can result from slant's being taken into account, ${ }^{1}$ familiarity may well operate also.

A correction takes place for another distortion in proximal stimulation that results from slanted viewing. When one looks at the picture of a tridimensional object from an oblique direction, its projection on the retina is distorted. If, for instance, one's vantage point is displaced to one side so that the viewing direction is horizontally slanted, horizontal distances in the picture are shortened in the retinal projection. In spite of such foreshortening, tridimensional shapes in pictures are often correctly perceived, but whether this is the result of compensation or of familiarity is not known. ${ }^{2}$ To resolve this issue, we tried to demonstrate that a compensation process operates that takes the angle between the picture plane and the line of sight into account. This is best done by providing deceptive cues for the orientation of the picture plane. We did it by having the subject view the slanted picture through a frame that was oriented orthogonally to his or her line of sight. A tendency to see the picture in the plane of the frame caused an apparent frontal orientation of the picture.

For the frame to have this effect, the subject had to observe monocularly. Special cues for the frame's orienta-

This work was supported by Grant BSN-8318772 from the National Science Foundation to Swarthmore College, Hans Wallach, principal investigator.

The authors' mailing address is: Department of Psychology, Swarthmore College, Swarthmore, PA 19801 tion were also provided. Dollar bills were attached to the frame, one at each side. In addition, a 15-cm-wide horizontal ledge running the length of the frame was attached along its bottom and was covered with a cloth, showing a checkerboard pattern that hung down $20 \mathrm{~cm}$ on three sides of the ledge and concealed the screen support behind it. This provided a potent cue for the frame's orientation. There was, of course, a control condition in which the frame was flush with the slanted picture and provided cues for its true orientation.

\section{EXPERIMENT 1}

\section{Method}

Subjects. Eighteen undergraduate students were paid for serving as subjects.

Equipment. A translucent screen, illuminated by a small light source from behind, served as the picture surface. The picture on the screen consisted of the shadow of a wire cube, also placed behind the screen. The wire cube was in the diamond position; a stem was perpendicularly attached to the midpoint of one of the edge wires so that it formed an angle of $135^{\circ}$ with each of the two adjacent cube faces. When the stem was vertical, two cube faces were vertical and formed diamonds. The cube was placed next to the screen in such a way that these diamond faces formed an angle of $45^{\circ}$ with the screen. The stem of the cube, which was given a small slant, formed an angle of $6^{\circ}$ with the screen. An edge of the cube measured $11.5 \mathrm{~cm}$.

The light source and the cube center were located on the same perpendicular to the screen. The distance of the light source from the screen was such that, had two cube faces been parallel to the screen, the shadow of one face would have been smaller by .93 than the shadow of the other face. Had a subject looked directly at that cube, from a distance of $159 \mathrm{~cm}$, it would have been given with a retinal projection of such a .93 distortion. We knew that correction for the distortion of polar perspective would have caused the subject to perceive a regular cube. Therefore, we selected $159 \mathrm{~cm}$ as the distance between the subject's eyes and the center of the cube shadow on the screen. The subject's seat and headrest were placed so that the subject's line of sight to the center of the cube shadow formed an angle of $60^{\circ}$ with the screen. The subject's eyes were at the level of the center of the cube shadow.

The frame and the patterned ledge were mounted on a vertical hinge and could be moved rapidly through an angle of $30^{\circ}$, from a position flush with the screen to a position orthogonal to the subject's line of sight. Also attached to the hinge was a shielded 7.5-W bulb that illuminated the frame and the pattern on the ledge. Ex- 
cept for this bulb and the light source behind the screen, which was a C-2R 6.2-V bulb, the room was dark. The frame was large enough to conceal the edges of the screen. Its aperture was $21.3 \mathrm{~cm}$ high and $24 \mathrm{~cm}$ wide.

Procedure. The subject was told that after being shown two figures in succession, he or she was to "judge whether one was a better cube than the other." The subject's left eye was occluded, and, while the experimenter changed the frame position, which took about $3 \mathrm{sec}$, the subject was asked to shut his or her eyes. The subject was instructed not to judge the figure while he or she saw it inverted. For half the subjects, the frame was initially flush with the screen; for the other half, it was initially in the frontal position.

\section{Results}

Sixteen of the 18 subjects judged the figure on the screen as more cube-like when the frame was slanted and provided veridical cues for the screen's orientation; 2 subjects gave the opposite report. Many subjects remarked spontaneously that the cube looked narrower when the frame was in the frontal orientation. Under that condition, the cube's apparent shape was more like the retinal projection of the shadow on the screen than like the shadow itself. Since the screen formed an angle of $60^{\circ}$ with the subject's line of sight, the width of the retinal projection of the shadow was smaller than the width of the shadów by $13.4 \%$. If constancy prevailed in the control condition, in which the orientation of the frame provided cues for the orientation of the picture plane, compensation for this $13.4 \%$ distortion should result in the perception of a more regular cube. When, on the other hand, the frontal frame obliterated the cues for the slant of the picture plane, the perceived cube should resemble the shadow's retinal projection. Our next experiment was designed to measure the difference in perceived width that apparently underlies the result just reported. The subjects gave estimates of the width, the height, and the depth of the shape they saw on the screen.

\section{EXPERIMENT 2}

\section{Method}

Subjects. Twelve undergraduate students were paid for serving as subjects.

Stimuli. The arrangements for this experiment were the same as in Experiment 1 except as follows: To make it easier for the subject to give the three size estimates, a smaller cube, with an edge $5.2 \mathrm{~cm}$ long, was used and the orientation of the cube in relation to the screen was changed to provide a more suitable shadow. The cube's two vertical faces were now nearly parallel to the screen, forming horizontal angles of only $10^{\circ}$ with it. This $10^{\circ}$ slant had little effect; it shortened the width of the shadow of the cube by only $2 \%$. The slant of the viewing direction, the angle it formed with the perpendicular to the picture plane, was increased from $30^{\circ}$ to $35^{\circ}$, causing the width of the retinal projection of the cube shadow to be diminished by $18 \%$. The movement of the frame was, of course, also increased to $35^{\circ}$. Finally, by making the width of the aperture smaller when the frame was frontally oriented, we compensated for the finding of Experiment 1 that when the frame was moved from the slanted to the frontal orientation, the retinal projection of the width of its aperture became larger.

The distance of the light source from the screen and the observation distance remained unchanged, but since the cube was now smaller, the distortion of the cube shadow due to the finite distance of the light source was also smaller; it now amounted to .97 .

Procedure. The subject was given outside calipers and was asked to set them so that the gap between the tips represented the horizontal or the vertical diagonal of the designated face of the cube-like figure. A similar estimate of the apparent distance between the two vertical faces was also obtained. These depth estimates were always made before the estimates of the horizontal and vertical diagonals. Half of the subjects started by giving the three estimates with the frame in the frontal orientation; the other half started with the frame flush with the screen. Half of each group of 6 subjects gave estimates for the horizontal diagonals first and for the vertical diagonals second; for the other 3 subjects that order was reversed. When a subject had given all six estimates, the whole procedure was repeated in the same order. The average of the two estimates obtained under the same condition became the subject's score.

\section{Results}

The results for the experimental condition in which the frame was in the frontal orientation are presented in the first column of Table 1 . They show that the perceived shape of a cube face was nearly the shape of its retinal projection. The mean estimate for the horizontal diagonal, that is, for the width of the cube face, was $5.53 \mathrm{~cm}$; the mean estimate for the vertical diagonal, its height, was $6.81 \mathrm{~cm}$. Thus, the apparent width of the cube face was $18.9 \%$ smaller than its apparent height. In the retinal projection of the cube face, this difference amounted to $20 \%$. This value represented an $18 \%$ loss in width that resulted from a $35^{\circ}$ slant in the viewing direction and a $2 \%$ loss in the width of the cube shadow itself. The latter was due to the $10^{\circ}$ angle that the cube face formed with the screen on which the shadow was formed. The $1.1 \%$ difference between the perceived proportions of the cube face and the proportions of its retinal projection was probably the result of the vertical-horizontal illusion. ${ }^{3}$ If that interpretation is accepted, it can be concluded that the perceived shape of the cube face was based on the shape of the retinal projection of that shape.

The results for the control condition, in which the frame had the same orientation as the screen and provided veridical cues for the screen's slant, are listed in the second column of Table 1 . They show a good degree of shape constancy. The mean estimate for the horizontal diagonal was $6.03 \mathrm{~cm}, 8 \%$ smaller than the mean estimate for the vertical diagonal, which was $6.56 \mathrm{~cm}$. Of this $8 \%$ difference, $2 \%$ resulted from the diminished width of the cube shadow mentioned above and $1 \%$ was probably due to the vertical-horizontal illusion. The remaining 5\%

Table 1

Mean Estimates (in Centimeters) of the Depth of a $5.2-\mathrm{cm}$

Wire Cube, of the Horizontal and Vertical Diagonals of One of Its Faces, and the Ratio of the Latter Two, with Standard Deviations of the Means $(N=12)$

\begin{tabular}{lccc}
\hline & Frame Frontal & Frame Flush \\
\hline Depth & $3.17(1.43)$ & $3.30(1.66)$ \\
Horizontal Diagonal & $5.53(1.36)$ & $6.03(1.48)$ \\
Vertical Diagonal & $6.81(1.69)$ & $6.56(1.58)$ \\
Ratio & .816 & .924 \\
\hline
\end{tabular}


represents the shortfall in a constancy process that tends to compensate for a distortion in retinal projection that resulted in horizontal distances' being diminished by $18 \%$.

This good degree of constancy can be compared with findings of the experimental condition, in which the cues provided by the frame in frontal orientation were fully effective and caused the retinal projection of the cube face to result in its perceived shape. For statistical purposes, the results of the experimental and the control condition can be compared by computing the ratios of individual subjects' width to height estimates for each condition. The means of these ratios, which are also listed in Table 1, are significantly different from each other $[t(11)=4.35$, $p<.002]$.

The mean depth estimates were about the same for the two conditions, but they fell short of the value expected had the subjects perceived the tridimensional shape as being as deep as a length of a side of a face. Based on the average of the mean estimates for the vertical diameter, which amounted to $6.69 \mathrm{~cm}$, the expected depth would have been $6.69 \times .707=4.73 \mathrm{~cm}$. The average of the two depth estimates was, in fact, only $3.24 \mathrm{~cm}$, more than $30 \%$ smaller. This shortfall was most likely the result of the paucity of cues for depth in the pictorial presentation of the cube.

\section{SUMMARY AND DISCUSSION}

In our experiments, a picture plane was slanted relative to the subject's line of sight and caused horizontal distances within the retinal projection of the picture to be shortened. A frame provided the cues for the orientation of the picture plane. When the frame had the same slant as the picture and provided cues for its true slant, a partial correction for the distortion in the retinal projection of the picture took place. But when the frame was in frontoparallel orientation and provided cues for the picture's being in that orientation, no correction took place. The perceived shape resembled the retinal projection of the shape in the picture. These results show that a compensation process that takes the slant of the picture plane into account can operate here.

This compensation process takes place only in picture perception. If the observer faced the real scene that was shown in the picture and changed the angle of view, the retinal projection of the scene would not show the distortion of horizontal distances; it would merely represent a somewhat different arrangement, in keeping with the changed angle of view. Does that mean that the compensation we demonstrated is a specific feature of picture perception? We believe so, although it could be argued that the compensation we demonstrated in Experiment 2 was not different from ordinary shape constancy, in which compensation for the distortion of the retinal projection of a surface slanted to the line of sight takes place. In fact, our Experiment 2 somewhat resembled the experiment by Wallach and Moore (1962), which demonstrated ordinary compensation for the effect of surface slant. But in Experiment 1 , compensation corrected truly for the distortion in the image of a tridimensional shape. To interpret this result as compensation for the effect of surface slant would require postulating a two-stage process. Compensation for the distortion of the retinal projection due to the slant of the picture plane would take place first and result in a representation of the picture structured as if it were seen in perpendicular view. This stage would be followed by the configurational process that constructs the perceived tridimensional scene from the corrected representation of the retinal projection. That that is actually the course of events is unlikely. Wallach and Slaughter (1986) have found evidence of picture perception with its own rules. Since perception of tridimensional shapes in pictures involves corrections that would not be needed in perception of the depicted arrangements if they were given in real tridimensional space, shape constancy in the perception of tridimensional shapes in pictures is likely to be governed by its own rules.

\section{REFERENCES}

Hochberg, J. (1971). Space and movement. In J. W. Kling \& L. A. Riggs (Eds.), Experimental psychology. New York, NY: Holt, Rinehart and Winston.

Perkins, D. N. (1973). Compensating for distortion in viewing pictures obliquely. Perception \& Psychophysics, 14, 13-18.

RosINSKI, R. R., \& FARMER, J. (1980). Compensation for viewing point in the perception of pictured space. In M. A. Hagen (Ed.), The perception of pictures (Vol. 1). New York: Academic Press.

Wallach, H., \& Moore, M. E. (1962). The role of slant in the perception of shape. American Journal of Psychology, 75, 289-293.

Wallach, H., \&laughter, V. (1986). On picture perception. Manuscript in preparation.

\section{NO'TES}

1. Several studies demonstrate perceptual correction for the effect of slant when slant is given stereoscopically. Whether such instances are a matter of compensation or simply of tridimensional shape perception may be debatable. However, Wallach and Moore (1962) demonstrated compensation in a monocularly viewed triangular shape.

2. Perkins (1973) demonstrated correction for the distortion of pictures of tridimensional shapes when they were viewed obliquely and ascribed it to compensation, but it appears that familiarity could also account for his results. See Rosinski and Farber (1980, p. 157) for a discussion of this point.

3. We measured the vertical-horizontal illusion to which the cube shadow was subject by presenting it on a frontoparallel screen, and obtained, from 9 subjects, size estimates for the vertical and horizontal diagonals of a cube face. The ratio of these estimates was .971 $(\mathrm{SD}=.062)$, while the actual ratio in the shadow was .98 . Hence, the illusion amounted to approximately $1 \%$.

(Manuscript received August 30, 1985; revision accepted for publication March 24, 1986.) 